\title{
Influence of TCSC on social welfare and spot price - A comparative study of PSO with classical method
}

\author{
S. K. Joshi ${ }^{1 *}$, K. S. Pandya ${ }^{2}$ \\ ${ }^{1 *}$ Electrical Engineering Department,,The M.S.University of Baroda, Vadodara,INDIA \\ ${ }^{2}$ Electrical Engineering Department, Charotar Institute of Technology, Changa, INDIA \\ "Corresponding Author: e-mail: skjoshi@ieee.org, Tel +91-265-2352958, Fax.+91-265-2423898
}

\begin{abstract}
In this paper, Particle Swarm Optimization (PSO) based algorithm has been suggested to find optimal location and setting of Thyristor Controlled Series Compensator (TCSC) to maximize Social Benefit (SB), considering its installation cost in competitive electricity market. PSO has simultaneously optimized generators' active powers output, generators' bus voltages, TCSC reactance and its location. In addition, the influence of optimally located TCSC on the magnitude of real power spot price, reactive power spot price, wheeling charges and bilateral transaction matrix has been investigated. The effectiveness of the proposed approach has been tested on IEEE 6 bus system and results obtained are compared with those obtained from various classical methods.
\end{abstract}

Keywords: Bilateral transaction, Particle Swarm Optimization, Social Benefit, Spot price

\section{Introduction}

In a last decade, many monopolistic electricity companies and electricity boards have been transformed into competitive structure to obtain higher efficiency, revenue, better service to consumers, to increase stability and much more. But, rapid rise in power demand, competition, outage and scare natural resources are some factors due to which transmission systems operate very near to their thermal limits. But because of economic, environmental and political reasons it is not preferable to build new transmission lines. So there is an interest in better utilization of existing capacities of power system by installing Flexible A.C. Transmission System (FACTS) device such as Thyristor Controlled Series Compensator (TCSC)(Hingorani et al, 2000). It is the power electronics based device which can enhance Total Transfer Capability, voltage stability, loadability, security etc. and can reduce losses, cost of generation, can remove congestion and fulfill transaction requirement rapidly, dynamically and efficiently.

Because of following reasons it is important to "optimally" locate TCSC in order to obtain its full benefits. (1) It is a costly device; (2) It has been proved that TCSC may have negative effect on system stability unless it is optimally placed (Yu et al, 2003); (3) It may adversely affect some market participants in deregulated power system.

\subsection{Literature Review:}

Various classical and artificial intelligence methods have been suggested to optimally locate FACTS devices with different kinds of objective functions.

Interior point method was used for system expansion with UPFC to maximize social welfare and to manage congestion (Lin et al, 2000). Genetic algorithm was used for multi-type FACTS placement to enhance system loadability. However, number of devices to be installed was assigned before optimization and installation cost was ignored (Gerbex et al, 2001). Mixed Integer Linear Programming was used to find optimum number, locations and setting of Thyristor Control Phase Shifting Transformer (TCPST) to optimize loadability and investment with respect to dc load flow model (Lima et al, 2003). Mixed Integer Non-linear programming was used for optimal location of FACTS to maximize social welfare based on multiple time periods. But it ignored the impact of FACTS on reactive power flow (Yu et al, 2004). Predictor corrector primal duel interior point linear programming was used to enhance Available Transfer Capability (ATC) using various FACTS devices. However, it did not optimize their 
ratings and locations (Xiao et al, 2003). A hybrid evolutionary algorithm was proposed to optimally place multi-type FACTS devices to maximize TTC and minimize losses (Jirapong et al, 2007). A sensitivity based approach was used to study the impact of Unified Power Flow Controller (UPFC) on real and reactive power spot prices (Verma et al, 2006). Sequential quadratic programming was used to study impact of TCSC on congestion and spot price. But it did not optimize TCSC setting and location (Archarya et al, 2007). A Mixed-Integer Quadratic programming was used to find optimal location of TCSC for enhancing system loadability, voltage profile considering its investment cost (Yang et al, 2007). A real genetic algorithm was proposed to optimally locate two TCSC for enhancing ATC and to get best voltage profile But generators' active output powers and bus voltages were not optimized (Rashidinejad et al, 2008). A mixed integer nonlinear programming was used to locate UPFC to maximize system loadability for pool and bilateral electricity markets (Kumar et al, 2009). A reactive power spot price index was proposed to place SVC to minimize total generation cost and to maximize loading margin (Singh et al, 2007).

So, it is revealed that most of the OPF problems are non-linear and non-convex. With the inclusion of FACTS control variables, they become even more nonlinear because they change the size of bus admittance matrix and dimension of the problem. Conventional classical optimization methods like gradient method, lamda iteration, linear programming etc. rely on the convexity assumption of objective function. They fail to capture discontinuities of objective function and may get trapped into local minima or diverge at all. Choice of initial starting point also affects the quality of solution. Also, they could find only single optimized solution in a single simulation run.

Thus, to find global optimum solution is a challenging task in optimization problem incorporating FACTS devices. To solve such problem, an artificial intelligent method called Particle Swarm Optimization may be used as it is a fast method and it provides global or near global solution (Kennedy et al, 1995). PSO has shown its superiority over other classical and AI methods with respect to execution time and global solution in solving economic dispatch problem (Park et al, 2005), optimal reactive power dispatch problem (Zhao et al, 2005)and congestion management (Dutta et al, 2008)

So in this paper, PSO based algorithm has been suggested to find the best location and setting of TCSC to maximize social benefit and to minimize total generation cost while satisfying various constraints of IEEE 6 bus test system. The same algorithm can be modified to maximize social benefit without considering TCSC. Comparisons are carried out with solutions obtained from Non-linear programming method (MINOS) (Murtagh et al) used in MATPOWER (Zimmerman et al, 2007). Transmission service pricing is also an important issue of deregulated market. Out of many suggested pricing methods, Locational Marginal Pricing (LMP) method is popular because it considers all system constraints and losses. As PSO cannot provide Lagrange multipliers which are required for finding LMP, an interior point method is used to calculate LMP. But choice of initial starting points greatly affects the quality of solution of interior point method (Xie et al, 2001). So in this paper, optimized output obtained from PSO are used as starting points and results are compared with default setting of Primal Dual Interior Point Method (Wang et al, 2007)used in MATPOWER. Lastly, influence of optimally placed TCSC by PSO on wheeling charges and secure bilateral transaction matrix has been studied and results are compared with those obtained by Primal Dual Interior Point Method.

This paper can be broadly divided into two parts. Firstly, PSO's superiority over various classical methods like non-linear programming method and interior point method in obtaining global solution without using TCSC has been searched out. Secondly, impact of optimally placed TCSC by PSO on social benefit, total generation cost, losses, real power and reactive power spot prices, wheeling charges and secure bilateral transaction has been investigated.

This paper has been organized as follows: Section 2 describes static modeling of TCSC. Section 3 explains overview of PSO method. Section 4 explains the optimal power flow formulation and major steps involved in the algorithm. Section 5 explains the PSO based algorithm in detail. Section 6 discusses simulation results and in Section 7, conclusive remarks have been given.

\section{Static modeling of TCSC}

As shown in Figure 1., the TCSC has been represented by a variable capacitive/inductive reactance inserted in series with the transmission line (Sharma, 2006). So the reactance of the transmission line is adjusted by TCSC directly. Let, $X_{m n}$ is the reactance of the transmission line, $X_{C}$ is the reactance of TCSC and $X_{\text {new }}$ is the new reactance of the line after placing TCSC between bus $\mathrm{m}$ and $\mathrm{n}$. Mathematically, equation is written as:

$X_{\text {new }}=X_{m n}-X c$

The modified power flow equations of the transmission line in the presence of TCSC are given as below:

$P_{m n}=V_{m}^{2} G_{m n}-V_{m} V_{n}\left(G_{m n} \cos \delta_{m n}+B_{m n} \sin \delta_{m n}\right)$

$Q_{m n}=-V_{m}^{2}\left(B_{m n}+\frac{B}{2}\right)-V_{m} V_{n}\left(G_{m n} \sin \delta_{m n}-B_{m n} \cos \delta_{m n}\right)$

$P_{n m}=V_{n}^{2} G_{m n}-V_{m} V_{n}\left(G_{m n} \cos \delta_{m n}-B_{m n} \sin \delta_{m n}\right)$

$Q_{n m}=-V_{n}^{2}\left(B_{m n}+\frac{B}{2}\right)+V_{m} V_{n}\left(G_{m n} \sin \delta_{m n}+B_{m n} \cos \delta_{m n}\right)$ 


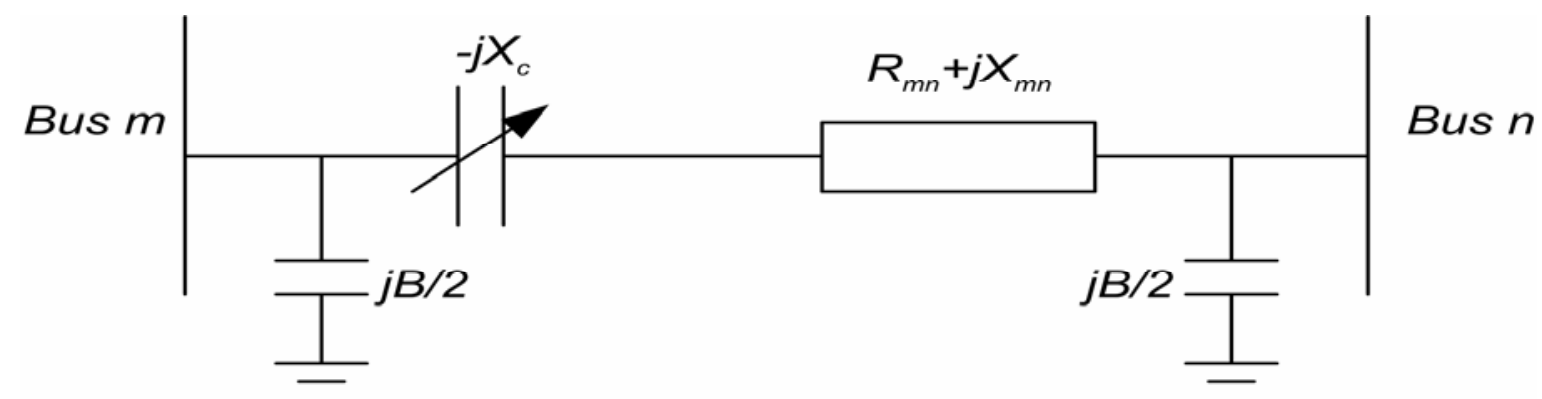

Figure 1. Equivalent circuit of transmission line after placing TCSC

where,

$G_{m n}=\frac{R_{m n}}{R_{m n}{ }^{2}+\left(X_{m n}-X_{c}\right)^{2}}, B_{m n}=\frac{-\left(X_{m n}-X_{c}\right)}{R_{m n}{ }^{2}+\left(X_{m n}-X_{c}\right)^{2}}$

$P_{m n}, Q_{m n}$ : Active and reactive power flow from bus $\mathrm{m}$ to $\mathrm{n}$

$P_{n m}, Q_{n m}$ : Active and reactive power flow from bus $\mathrm{n}$ to $\mathrm{m}$

$G_{m n}$ : New line conductance between bus $\mathrm{m}$ and $\mathrm{n}$

$B_{m n}$ : New line susceptance between bus $\mathrm{m}$ and $\mathrm{n}$

$R_{m n}:$ Line resistance between bus $\mathrm{m}$ and $\mathrm{n}$

\section{Basics of the Particle Swarm Optimization}

PSO is a fast, simple and efficient population-based optimization method which was proposed by Eberhart and Kennedy. It has been motivated by the behavior of organisms such as fish schooling and bird flocking. In PSO, a "Swarm" consists of number of particles which represent the possible solutions. The coordinates of each particle is associated with two vectors, namely the position $\left(x_{i}\right)$ and velocity $\left(v_{i}\right)$ vectors. The size of both vectors is same as that of the problem space dimension. All particles in a swarm fly in the search space to explore optimal solutions. Each particle updates its position based upon its own best position, global best position among particles and its previous velocity vector according to the following equations:

$v_{i}^{k+1}=w \times v_{i}^{k}+c_{1} \times r_{1} \times\left(p_{\text {best }_{i}}-x_{i}^{k}\right)+c_{2} \times r_{2} \times\left(g_{\text {best }}-x_{i}^{k}\right)$

$x_{i}^{k+1}=x_{i}^{k}+\chi \times v_{i}^{k+1}$

where,

$v_{i}^{k+1}$ : The velocity of $i^{\text {th }}$ particle at $(k+1)^{\text {th }}$ iteration

$w$ : Inertia weight of the particle

$v_{i}^{k}$ : The velocity of $i^{\text {th }}$ particle at $k^{\text {th }}$ iteration

$c_{1}, c_{2}$ : Positive constants having values between $[0,2.5]$

$r_{1}, r_{2}$ : Randomly generated numbers between $[0,1]$

$p_{\text {best }}$ : The best position of the $i^{\text {th }}$ particle obtained based upon its own experience

$g_{\text {best }}:$ Global best position of the particle in the population

$x_{i}^{k+1}$ : The position of $i^{\text {th }}$ particle at $(k+1)^{\text {th }}$ iteration

$x_{i}^{k} \quad$ : The position of $i^{\text {th }}$ particle at $k^{\text {th }}$ iteration

$\chi$ : Constriction factor. It may help insure convergence. Its low value facilitates fast convergence and little exploration while high value results in slow convergence and much exploration.

If no restriction is imposed on the maximum velocity $\left(v_{\max }\right)$ of the particles then there is likelihood that particles may leave the search space. So velocity of each particle is controlled between $\left(-v_{\max }\right)$ to $\left(v_{\max }\right)$.

Suitable selection of inertia weight $w$ provides good balance between global and local explorations. It is set according the following equation.

$w=w_{\max }-\frac{w_{\max }-w_{\min }}{i t e r_{\max }} \times$ iter 
Where, $w_{\max }$ is the value of inertia weight at the beginning of iterations, $w_{\min }$ is the value of inertia weight at the end of iterations, iter is the current iteration number and iter $_{\max }$ is the maximum number of iterations.

\section{Proposed solution}

The proposed algorithm is given in section 5. The major steps involved in the algorithm are summarized in the following.

\subsection{Initial population}

In this paper, a particle is made of continuous and integer control variables. The continuous variables include the generators' active powers output $\left(\mathrm{PG}_{2}, \ldots, \mathrm{PG}_{\mathrm{NG}}\right)$, generators' bus voltages $\left(\mathrm{VG}_{1}, \ldots, \mathrm{VG}_{\mathrm{NG}}\right.$ )and reactance value of TCSC (X). Generators' active powers and generators' bus voltages are generated randomly within their permissible minimum and maximum limits. Reactance of TCSC is generated randomly between 0 and 1(normalized form, $\left.X_{(N)}\right)$ and its actual value $\left(X_{(D)}\right)$ is found using eqn. (9).

$X_{(D)}=X_{(\min )}+\left(X_{\max }-X_{\min }\right) \times X_{(N)}$.

Where, $X_{\text {min }}$ and $X_{\text {max }}$ are minimum and maximum values of the variable.

The integer variable consists of possible location (Loc) of TCSC. The particles can be generated in matrix form as shown in Figure 2.

\begin{tabular}{|c|c|c|c|c|c|c|c|c|}
\hline Particle No. & $\mathrm{PG}_{2}$ & $\ldots$ & $\mathrm{PG}_{\mathrm{NG}}$ & $\mathrm{VG}_{1}$ & $\ldots$ & $\mathrm{VG}_{\mathrm{NG}}$ & $\mathrm{X}_{\mathrm{C}}$ & $\mathrm{LOC}$ \\
\hline 1 & $\mathrm{PG}_{2,1}$ & $\ldots$ & $\mathrm{PG}_{\mathrm{NG}, 1}$ & $\mathrm{VG}_{1,1}$ & $\ldots$ & $\mathrm{VG}_{\mathrm{NG}, 1}$ & $\mathrm{X}_{\mathrm{C} 1}$ & $\mathrm{LOC}_{1}$ \\
\hline 2 & $\mathrm{PG}_{2,2}$ & $\ldots$ & $\mathrm{PG}_{\mathrm{NG}, 2}$ & $\mathrm{VG}_{1,2}$ & $\ldots$ & $\mathrm{VG}_{\mathrm{NG}, 2}$ & $\mathrm{X}_{\mathrm{C} 2}$ & $\mathrm{LOC}_{2}$ \\
\hline$\ldots$ & $\ldots$ & $\ldots$ & $\ldots$ & $\ldots$ & $\ldots$ & $\ldots$ & $\ldots$ & $\ldots$ \\
\hline $\mathrm{i}$ & $\mathrm{PG}_{2, \mathrm{i}}$ & $\ldots$ & $\mathrm{PG}_{\mathrm{NG}, \mathrm{i}}$ & $\mathrm{VG}_{1, \mathrm{i}}$ & $\ldots$ & $\mathrm{VG}_{\mathrm{NG}, \mathrm{i}}$ & $\mathrm{X}_{\mathrm{Ci}}$ & $\mathrm{Loc}_{\mathrm{i}}$ \\
\hline
\end{tabular}

Figure 2. Representation of particle

Where, $\mathrm{PG}_{2, \mathrm{i}}, \mathrm{PG}_{\mathrm{NG}, \mathrm{i}}$ : From $2^{\text {nd }}$ to $\mathrm{NG}^{\text {th }}$ generators' active output powers corresponding to $\mathrm{i}^{\text {th }}$ particle excluding slack bus generator power.

$\mathrm{VG}_{1, \mathrm{i}}, \mathrm{VG}_{\mathrm{NG}, \mathrm{i}}$ : From $1^{\text {st }}$ to $\mathrm{NG}^{\text {th }}$ generators' voltage magnitudes corresponding to $\mathrm{i}^{\text {th }}$ particle including slack bus generator voltage.

$\mathrm{X}_{\mathrm{Ci}}$ : Reactance of TCSC corresponding to $\mathrm{i}^{\text {th }}$ particle

Loc $_{\mathrm{i}}$ : Location (line number) of TCSC corresponding to $i^{\text {th }}$ particle.

If TCSC is not included in the transmission system, then variables $\mathrm{X}_{\mathrm{c}}$ and Loc are not considered.

If there are total $\mathrm{i}$ number of particles and if each particle consists of $\mathrm{j}$ number of control variables, then the dimension of a population becomes $\mathrm{i} * \mathrm{j}$.

\subsection{Installation of TCSC.}

After generating initial population of particles, for each particle TCSC is randomly installed in the transmission line with randomly generated reactance. Then after, new value of bus admittance matrix is found out.

\subsection{Power flow}

Run full a.c. Newton-Raphson power flow for all particles to obtain generators' active and reactive output powers, bus voltages, load angles, line flows, active and reactive power losses of transmission lines.

\subsection{Optimal power flow problem formulation}

An OPF (fitness function) to maximize Social Benefit (to minimize total generation cost) considering installation cost of TCSC subject to various equality and inequality constraints using PSO can be formulated as (10).

$\min \left\{\sum_{m=1}^{N_{G}} C_{G m}\left(P_{G m}\right)-\sum_{n=1}^{N_{D}} B_{D n}\left(P_{D n}\right)+\left(I C_{T C S C}\right)+(P F)\right\}$ 
An OPF for maximizing social benefit without considering TCSC can be formulated as (11)

$\min \left\{\sum_{m=1}^{N_{G}} C_{G m}\left(P_{G m}\right)-\sum_{n=1}^{N_{D}} B_{D n}\left(P_{D n}\right)+(P F)\right\}$

Subject to the power balance equations (equality constraints)

$\left\{\begin{array}{l}P_{G m}-P_{D m}-\sum_{n=1}^{N_{b}}\left|V_{m}\right|\left|V_{n}\right|\left|Y_{m n}\right| \cos \left(\delta_{m}-\delta_{n}-\theta_{m n}\right)=0 \\ Q_{G m}-Q_{D m}-\sum_{n=1}^{N_{b}}\left|V_{m}\right|\left|V_{n}\right|\left|Y_{m n}\right| \sin \left(\delta_{m}-\delta_{n}-\theta_{m n}\right)=0\end{array}\right\}$

Various operating constraints (inequality constraints)

$$
\begin{aligned}
& P_{G m}^{\min } \leq P_{G m} \leq P_{G m}^{\max }, m \forall N_{G} \\
& Q_{G m}^{\min } \leq Q_{G m} \leq Q_{G m}^{\max }, m \forall N_{G} \\
& \left|S_{l}\right| \leq S_{l}^{\max }, I \forall N_{L} \\
& V_{m}^{\min } \leq V_{m} \leq V_{m}^{\max }, m \forall N_{b} \\
& X_{C}^{\min } \leq X_{C} \leq X_{C}^{\max } \text { p.u. }
\end{aligned}
$$

Where,

$C_{G m}\left(P_{G m}\right)$ : Bid function of $\mathrm{m}^{\text {th }}$ generator bus (seller bus)

$B_{D n}\left(P_{D n}\right)$ : Bid function of $\mathrm{n}^{\text {th }}$ consumer bus (buyer bus)

$I C_{\text {TCSC }} \quad$ : Installation cost of TCSC (\$)

$P F \quad$ : Penalty Function

$P_{G m}, Q_{G m}$ : Active and reactive power generation at bus $m$

$P_{D m}, Q_{D m}$ : Active and reactive power demand at bus $\mathrm{m}$

$\left|V_{m}\right| \angle \delta_{m}$ : Complex voltage at bus $\mathrm{m}$

$\left|Y_{m n}\right| \angle \theta_{m n}: \mathrm{mn}^{\text {th }}$ element of bus admittance matrix

$P_{G m}^{\min }, P_{G m}^{\max }$ : Active power generation limits at bus $\mathrm{m}$

$Q_{G m}^{\min }, Q_{G m}^{\max }$ : Reactive power generation limits at bus $\mathrm{m}$

$S_{l}^{\max }$ : Thermal limit of $l^{\text {th }}$ transmission line

$V_{m}^{\min }, V_{m}^{\max }$ : Voltage magnitude limits at bus $\mathrm{m}$

$X_{C}^{\min }=-0.85 \times X_{m n}$ : Lower limit of reactance of TCSC

$X_{C}^{\max }=0.2 \times X_{m n}$ : Upper limit of reactance of TCSC

$N_{L}$ : Total number of transmission lines

$N_{b}$ : Total number of buses

$N_{G}$ : Total number of generator buses

$N_{D}$ : Total number of load buses

The cost function of TCSC is given in Siemens database and used in (Cai et al, 2004). Mathematically it is written as equ (18)

$C_{\text {TCSC }}=0.0015(S)^{2}-0.7130(S)+153.75$

Where, $C_{\text {TCSC }}$ is the cost of TCSC in US\$/KVAR and $S$ is the operating range of the TCSC in MVAR.

$$
S=\left|Q_{1}\right|-\left|Q_{2}\right|
$$

Where $Q_{1}$ is the reactive power flow in the line before placing TCSC in MVAR and $Q_{2}$ is the reactive power flow in the line after placing TCSC in MVAR.

The installation cost (\$) of TCSC is given by (20)

$I C_{T C S C}=C_{T C S C} \times S \times 1000$

Square penalty function is used to handle inequality constraints such as reactive power output of generator buses, voltage magnitude of all buses and transmission lines thermal limits respectively, as shown in (21) and (22).

$P F=k_{1} \times \sum_{m=1}^{N_{G}} f\left(Q_{G m}\right)+k_{2} \times \sum_{m=1}^{N_{b}} f\left(V_{m}\right)+k_{3} \times \sum_{m=1}^{N_{L}} f\left(S_{l m}\right)$ 
$f(x)=\left\{\begin{array}{ll}0 & \text { if } x^{\min } \leq x \leq x^{\max } \\ \left(x-x^{\max }\right)^{2} & \text { if } x>x^{\max } \\ \left(x^{\min }-x\right)^{2} & \text { if } x<x^{\min }\end{array}\right\}$

where,

$k_{1}, k_{2}, k_{3}$ : Penalty coefficients for reactive power output of generator buses $\left(Q_{G m}\right)$, voltage magnitude $\left(V_{m}\right)$ of all buses and transmission line loading $\left(S_{l m}\right)$, respectively. The value of each coefficient is equal to 1000 .

$x^{\min }, x^{\max }$ : Minimum and maximum limits of variable $\mathrm{x}$.

4.5 Spot Price

In the second phase of optimization, Real power spot price and Reactive power spot price (Locational Marginal Price) at each generator bus and load bus is found. But, PSO does not provide Lagrange multipliers (required for determination of spot price) during optimization. So an interior point method is used to calculate LMP. But choice of initial starting points greatly affects the quality of solution and convergence of interior point method. So in this work, optimized output obtained from PSO are used as starting points for interior point method and results are compared with those obtained by using default setting of Primal Dual Interior Point Method used in MATPOWER.

\subsection{Wheeling Charge}

Wheeling is the transmission of electrical energy from a seller bus to a buyer bus through a transmission network owned by a third party. Wheeling rates are the charges given to the transmission owner.

Wheeling rate $\left(W_{p}\right)$ for the real power can be expressed as (23)

$W_{p}=\lambda_{p B}-\lambda_{p S}$

Where, $\lambda_{p B}$ is the spot price of real power at the buyer bus-B,

$\lambda_{p S}$ is the spot price of real power at the seller bus-S

Wheeling charge for purchase of real power $\left(P_{B}\right)$ is expressed as (24)

$W C_{p}=P_{B} \times W_{p}$

Wheeling rate $\left(W_{q}\right)$ for the reactive power can be expressed as (25)

$W_{q}=\lambda_{q B}-\lambda_{q S}$

Where, $\lambda_{q B}$ is the spot price of reactive power at the buyer bus-B,

$\lambda_{q S}$ is the spot price of reactive power at the seller bus-S

Wheeling charge for purchase of reactive power $\left(Q_{B}\right)$ is expressed as (26)

$W C_{q}=Q_{B} \times W_{q}$

\subsection{Social Benefit of various market participants.}

Social Benefit was originally defined by the Bergson-Samuelson social welfare function and latter on modified by the economists to refer to the benefit of various participants (Luenberger, 1995). The benefit to the generator is the revenue minus the generation cost, referred to as the generator surplus (Producer surplus). It can be also expressed as (27), if the spot price at the generator bus is known. Transmission network is managed by a non-profit organization known as the Wholesaler( Merchandize). Different spot prices exist at the generator buses and load buses due to the losses and congestion. So merchandize surplus is the revenue obtained to wholesaler to compensate losses and congestion as given in (29). Consumer surplus is the benefit obtained to consumer from the consumption of electrical energy as given in (28). Social Benefit is the addition of Producer surplus, Merchandize surplus and Consumer surplus.

producer surplus $=\sum_{m=1}^{N_{G}} \frac{1}{2}\left(\lambda_{m}-b_{g m}\right)\left(P_{G m}-P_{G m}^{\min }\right)$

Where, $\lambda_{m}$ is the spot price at generator bus $\mathrm{m}$

$b_{g m}$ is the linear coefficient in the quadratic generator bid function

$P_{G m}$ is the real power output of generator $\mathrm{m}$ 
$P_{G m}^{\min }$ is minimum real power generation limit of generator $\mathrm{m}$

consumersurplus $=\sum_{n=1}^{N_{D}} \frac{1}{2}\left(b_{d n}-\lambda_{n}\right)\left(P_{D n}-P_{D n}^{\min }\right)$

Where, $b_{d n}$ is the linear coefficient in the quadratic demand function

$\lambda_{n}$ is the spot price at load bus $n$

$P_{D n}$ is the real power demand at load bus $\mathrm{n}$

$P_{D n}^{\min }$ is minimum real power demand limit at load bus $\mathrm{n}$

merchandize surplus $=\sum_{n=1}^{N_{D}} \lambda_{n} \times P_{D n}-\sum_{m=1}^{N_{G}} \lambda_{m} \times P_{G m}$

\subsection{Effect of TCSC on the magnitude of bilateral transactions}

A bilateral transaction is made directly between a seller and a buyer without any third party intervention. Mathematically, each bilateral transaction between a seller at bus $\mathrm{m}$ and buyer at bus $\mathrm{n}$ satisfies the following power balance relationship:

$P_{G m}-P_{D n}=0$

The secure bilateral transaction matrix is obtained from the proposed bilateral transaction matrix after obtaining social benefit.

$\min \sum_{m} \sum_{n}\left(T_{m n}-T_{m n}^{o}\right)^{2}$

Where $T_{m n}$ is the secure bilateral transaction between seller and buyer buses.

$T_{m n}^{o}$ is the proposed bilateral transaction between seller and buyer buses.

The value of secure bilateral transaction matrix has been obtained without and with optimally placed TCSC using PSO. Its value is also obtained by interior point method. Results obtained using PSO method is compared with those obtained from interior point method.

\section{Algorithm to optimally locate TCSC to maximize social benefit using PSO}

Step 1: Input the data of lines, generators, buses and loads. Choose population size of particles and convergence criterion. Define type of power transaction.

Step 2: Select generators' active power output excluding slack bus generator power, voltage magnitude of generator buses including slack bus voltage, reactance setting of TCSC and location (line number) of TCSC as control variables. If TCSC is not included in the OPF problem, then variables- reactance setting of TCSC and location are not considered.

Step 3: Randomly generate population of particles in such a way that their variables fall within their feasible limits.

Step 4: Modify the bus admittance matrix.

Step 5: Run full a.c. Newton-Raphson load flow to get line flows, active power generations, reactive power generations, line losses and voltage magnitude of all buses.

Step 6: Calculate the penalty function of each particle using eqn. (21).

Step 7: Calculate the fitness function of each particle using eqn. (10) (if TCSC is included in optimization) Or eqn. (11)(if TCSC is not included in optimization).

Step 8: Find out the "global best" $\left(g_{\text {best }}\right)$ particle having minimum value of fitness function in the whole population and "personal best" $\left(p_{\text {besti }}\right)$ of all particles.

Step 9: Generate new population using eqns. (6) and (7).

Step 10: Go to step 4 until maximum number of iterations are completed.

Step 11: After the optimization, the fitness value of $g_{\text {best }}$ particle gives minimized value of total generation cost (if the loads are inelastic) including installation cost of TCSC or gives maximized value of Social Benefit (if the loads are elastic). Coordinates of $g_{\text {best }}$ particle give the optimized values of generators' active power output, generators' bus voltages, optimal reactance setting of TCSC and location of TCSC respectively.

Step 12: Real power and reactive power spot price at each generator and load bus is found using interior point method with and without using TCSC. Optimized outputs obtained by PSO in step 11 are used as the starting point in interior point method.

Step 13: Wheeling charges for real power and reactive power can be calculated using eqn (24) and (26).

Step 14: Surplus of various market participants could be obtained using eqn (27), (28) and (29). 


\section{Simulation results and discussions}

The simulation studies were carried out on intel CORE 2 Duo, 2GB of RAM, $2.20 \mathrm{GH}_{\mathrm{Z}}$ system in MATLAB 7.6 platform. The IEEE 6 bus system has been used to test the proposed algorithm. The bus, line and generator data was taken from (Zimmerman et al, 2007). It consists of 3 generators and 11 transmission lines.

As shown in Table 1, generation cost minimization OPF is solved using PSO (Case 1A) and non-linear programming (MINOS solver, Case 1C) without using TCSC. The obtained total generation cost is 3,128.8 $\$ / \mathrm{h}$ by PSO, whereas it is 3,143.97 $\$ / \mathrm{h}$ by MINOS. It is due to that fact that PSO can simultaneously optimize generators' active powers output and generator bus voltages. So it can find a global solution. But MINOS trapped into local minima. Active power loss and reactive power loss obtained from PSO are lesser than those obtained from MINOS. Reactive power output of generators no. 2 and 3 are reduced by PSO, so they can be even more efficiently utilized. In case 1B, optimal reactance setting and location of TCSC are found by PSO to minimize the composite objective function which consists of total generation cost and installation cost of TCSC. Total generation cost is 3,125.3 $\$ / h$, which is lesser than those obtained in cases $1 \mathrm{~A}$ and 1C. Optimal installation cost of TCSC obtained was $0.3977^{*} 10^{6}(\$)$. Optimal reactance of TCSC was negative, means it operated in capacitive mode. Optimal location of TCSC was the line that was connected between bus 1 and bus 4. Also, Optimally placed TCSC significantly reduced active power losses and reactive power losses.

Table 1. Comparison of results of Cost minimization by PSO and MINOS

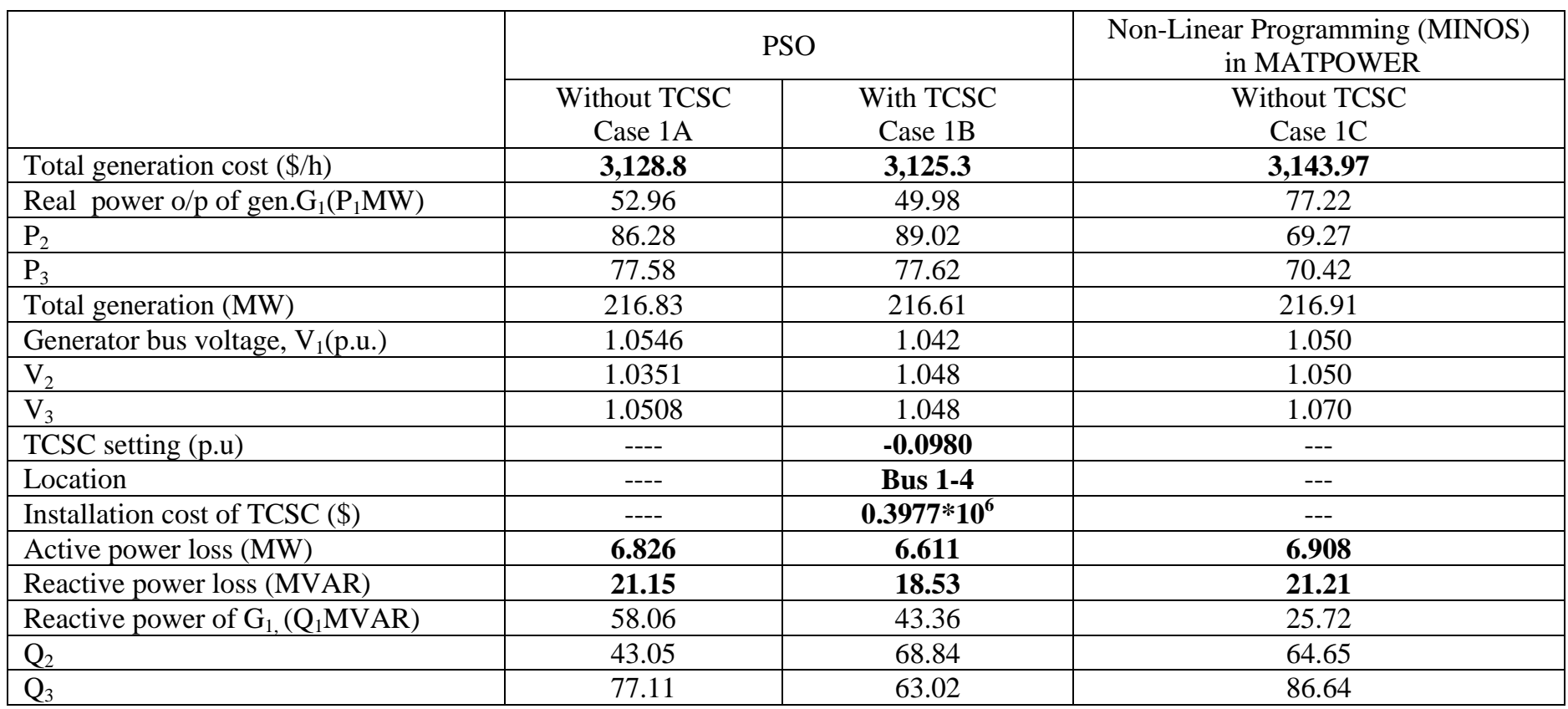

As shown in Figures 3(a) and 3(b), Real power spot price and Reactive power spot price (Locational Marginal Price) at each generator bus and load bus were found. The generators are connected at bus 1,2 and 3. The loads are connected at bus 4, 5 and 6 . The spot prices of real power are very high as compared to that of reactive power. Most of the spot prices obtained at load buses in Case 3A were lower than that of Case 3C. So it is cleared that consumers' surplus increase when PSO's optimized outputs are used as starting point for interior point method. Similarly, most of the spot prices obtained at generator buses in Case 3A were higher than that of Case 3C. So producers' surpluses increase when PSO's optimized results are used as starting point for IP method. Optimally placed TCSC (Case 3B) reduce real power spot price and reactive power spot price of all load buses and of some generator buses. It is because TCSC redistributes power flow in the transmission lines in such a way to decrease the losses and thus it tries to equalize spot prices at various load buses. Thus, TCSC increases consumers' surpluses and producers' surpluses. 
Case 3A- Optimized solutions of PSO used as starting points in PDIPM (Without TCSC)

Case 3B- Optimized solutions of PSO used as starting points in PDIPM. (With TCSC)

Case 3C- Default setting of Primal/dual Interior point method (PDIPM) in MATPOWER (Without TCSC)

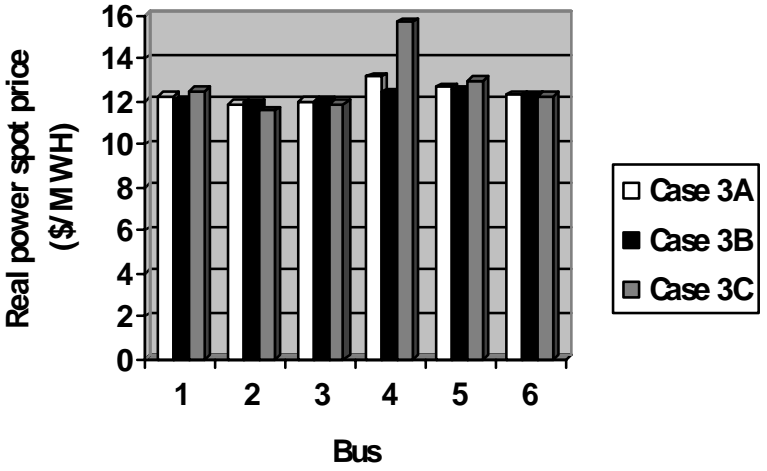

Figure 3(a). Comparison of real power spot price obtained from PSO based starting points and default starting points in interior point method.

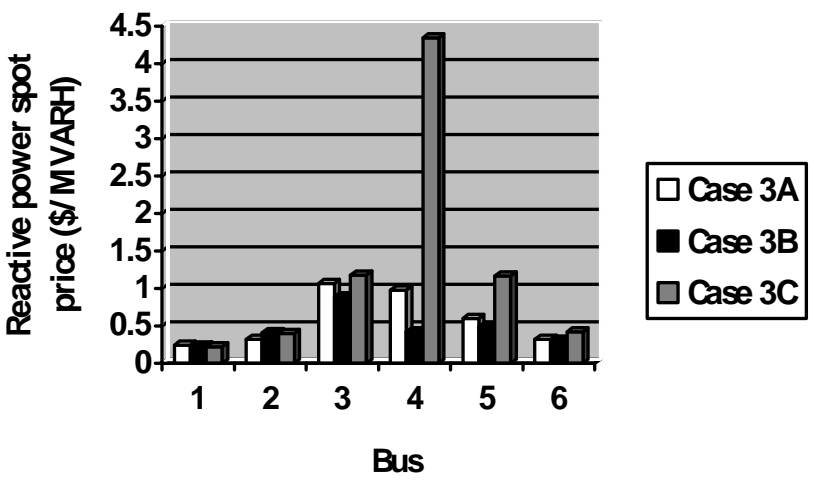

Figure 3(b). Comparison of reactive power spot price obtained from PSO based starting points and default starting points in interior point method.

Figure 4 shows the wheeling charges for real power of various bilateral transactions. Comparing the results of Case 4A with Case 4C reveals that most of the wheeling charges obtained by PSO have lesser value than those obtained by IP method. For transaction between buses 3-6 wheeling charge of Case 4A is greater than that of Case 4C, because load at bus 6 is responsible for large power flow in some transmission lines which increases its spot price. When TCSC is optimally placed (Case 4B) by PSO, it significantly reduces wheeling charges for real power. For transaction between bus 2-4, highest reduction in wheeling charge is obtained because the value of load at bus 4 is such that it requires less power flow in some transmission lines and TCSC reduces losses to a great extend and thus wheeling charge is significantly reduced.

Case 4A-Wheeling charges obtained by PSO (Without TCSC)

Case 4B- Wheeling charges obtained by PSO (With TCSC)

Case 4C-Wheeling charges obtained by Interior point method (Without TCSC)

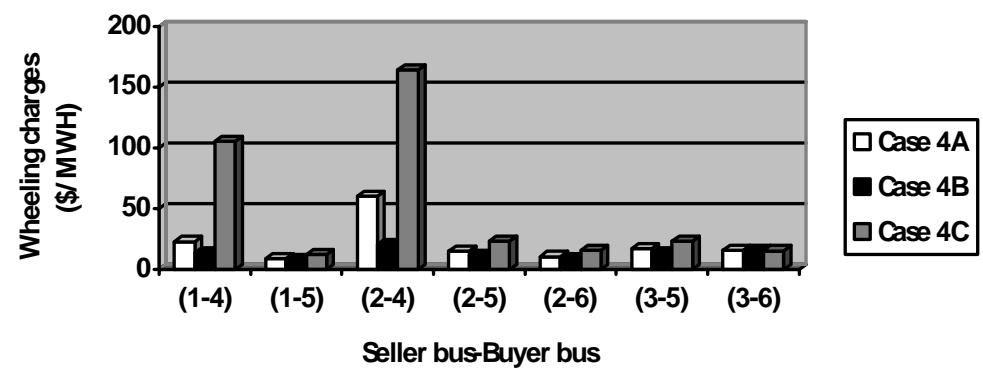

Figure 4. Wheeling charges $(\$ / \mathrm{MWH})$ of real power of selected bilateral transactions

Figure 5 shows the wheeling charges for reactive power of various bilateral transactions. It can be seen that even though the spot price of reactive power are very less as compared to that of real power spot price, the wheeling charge of reactive power is quite significant and should be considered while formatting the bilateral contracts. Wheeling charge of reactive power for transaction between buses 2-6 becomes negative after placing TCSC (Case 5B). So buyer bus 6 will get discount for purchasing reactive power from seller bus 2. So TCSC encourages buyer bus 6 to reschedule its load in such a way that it may even get more discounts from the transaction. 
Case 5A:Wheeling charges obtained by PSO (Without TCSC)

Case 5B:Wheeling charges obtained by PSO (With TCSC)

Case 5C:Wheeling charges obtained by Interior point method (Without TCSC)

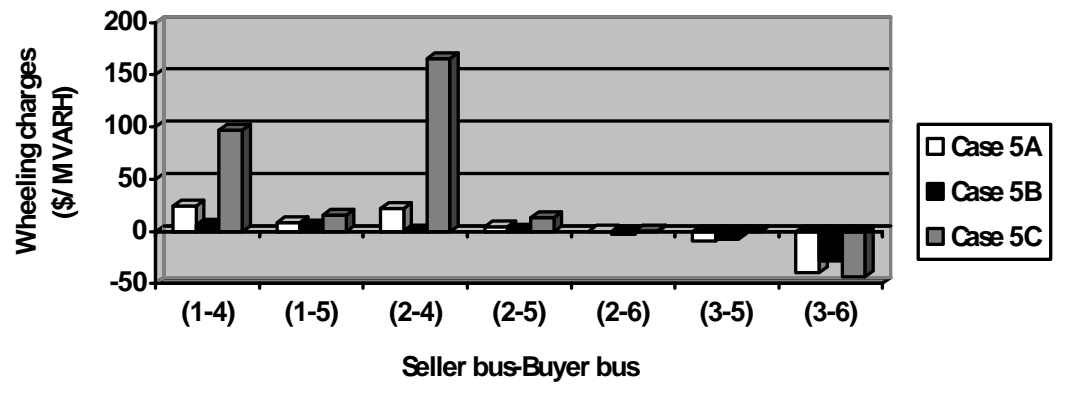

Figure 5. Wheeling charges (\$/MVARH) of reactive power of selected bilateral transactions

As shown in Figure 6, Consumers' surplus, Merchandize surplus and Producers' surplus can be obtained using eqn (28), (29) and (27) respectively. Optimally placed TCSC (Case 6B) can significantly increase Consumers' surplus and Producers' surplus, which are the main aims of deregulation. Merchandize surplus includes cost of losses and cost of congestion. As TCSC reduces losses of the transmission system and thus removes congestion, it drastically decreases Merchandize surplus.

Case 6A: Surplus obtained by PSO (Without TCSC)

Case 6B: Surplus obtained by PSO (With TCSC)

Case 6C: Surplus obtained by Interior point method (Without TCSC)

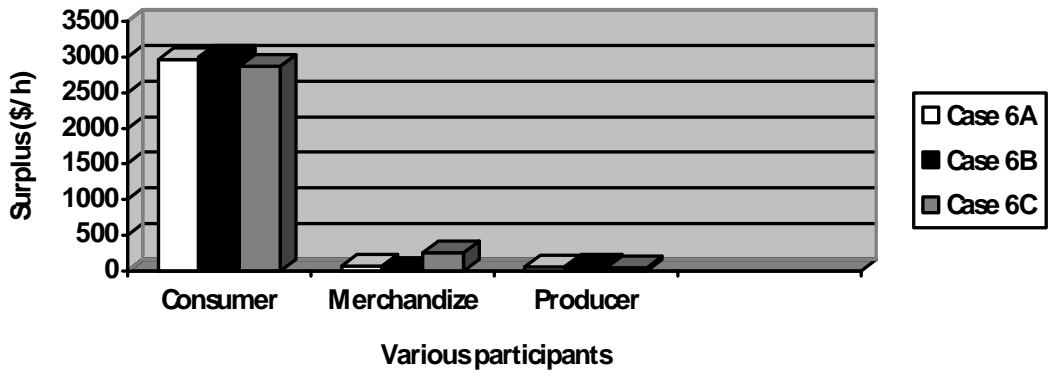

Figure 6. Comparison of surplus obtained by PSO and Interior point method

Figure 7 shows the proposed bilateral transaction matrix. The elements of the figure represent the bilateral contract between the $\mathrm{i}^{\text {th }}$ seller bus (generator bus) and $\mathrm{j}^{\text {th }}$ buyer bus (load bus). The elements in the table have positive real values. Some contracts between the generator bus and load bus have zero values. The secure bilateral transaction matrix is obtained from proposed bilateral transaction matrix after maximizing social benefit function.

Figure 8 shows the secure bilateral transaction matrix obtained by PSO and interior point method, without using TCSC. By comparing them, it was seen that PSO could fulfill more contractual demand than interior point method in most of the transactions. It was because PSO could simultaneously optimized generators' active power output and generators' bus voltages, whereas interior point method could not find the same. 


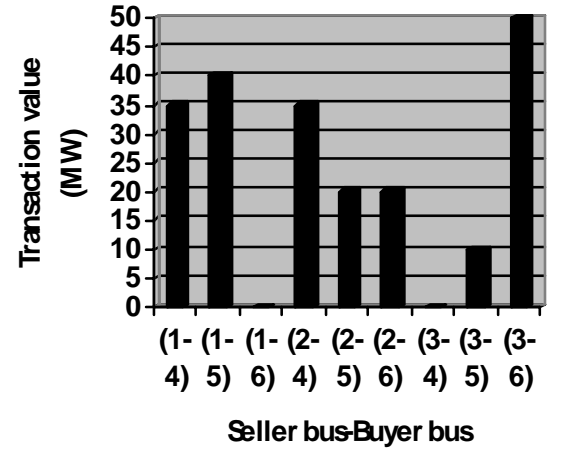

Figure 7. Proposed bilateral transaction matrix (without TCSC)

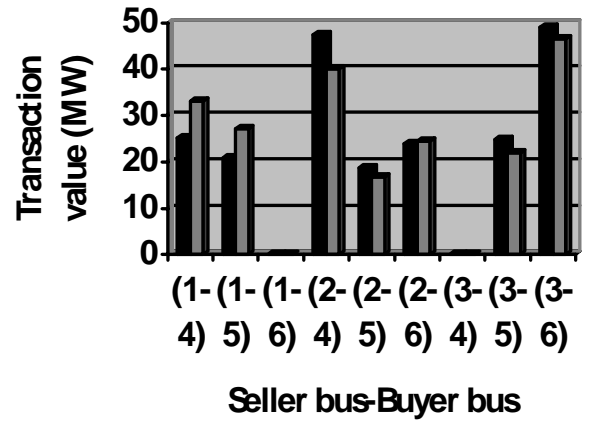

PSO $\square$ Interior point method

Figure 8. Comparison of secure bilateral transaction matrix obtained by PSO and Interior point method (without TCSC)

Figure 9 shows the impact of optimally placed TCSC by PSO on the magnitude of secure bilateral transaction matrix. Comparing the figures 7 and 9, it can be seen that the pattern of secure bilateral transactions is different and it is more uniform in the presence of TCSC. It is because of power flow control capability of TCSC.

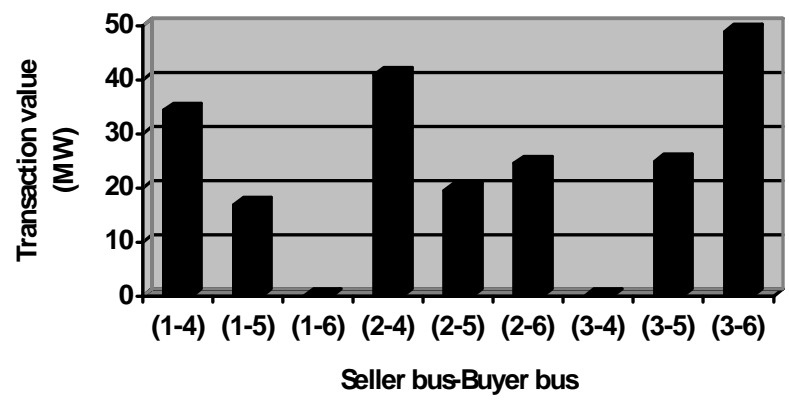

Figure 9. PSO based secure bilateral transaction matrix with TCSC

\subsection{Effects of PSO parameters on convergence}

PSO parameters such as cognitive parameter $\left(\mathrm{C}_{1}\right)$, social parameter $\left(\mathrm{C}_{2}\right)$, Constriction factor $(\chi)$, maximum inertia weight $\left(\mathrm{W}_{\max }\right)$, minimum inertia weight $\left(\mathrm{W}_{\min }\right)$, upper limit of velocity $\left(\mathrm{V}_{\max }\right)$, and lower limit of velocity $\left(-\mathrm{V}_{\max }\right)$ were selected through experiments and their effects on the value of optimal generation cost have been studied. The results are shown in Table 3 . The population of 35 particles was taken for all cases 3TA-3TE and 25 independent trials were carried out for each case. It was seen that case 3TC $\left(\mathrm{C}_{1}=1.2, \mathrm{C}_{2}=1, \chi=0.3, \mathrm{~W}_{\max }=0.7, \mathrm{~W}_{\min }=0.3, \mathrm{~V}_{\max }=0.25\right.$, and $\left.-\mathrm{V}_{\max }=-0.25\right)$ gave the minimum generation cost of 3,125.3 \$/h. To check the convergence characteristics of PSO with the selected parameters of case 3TC, number of simulations was carried out. Convergence criterion taken was 25 iterations. The variation in generation cost with the iteration number is shown in Figure 10 for PSO method and in Figure 11 for NLP (MINOS) method. Comparing both the figures, it is observed that PSO provides global solution in less than 15 iterations. Whereas, NLP was trapped into local minima.

Table 3. Effect of PSO parameters on convergence

\begin{tabular}{|c|l|c|c|c|c|}
\hline Case & $\mathrm{C}_{1}, \mathrm{C}_{2}$ & Constriction factor $(\chi)$ & $\mathrm{W}_{\max }, \mathrm{W}_{\min }$ & $\mathrm{V}_{\max },-\mathrm{V}_{\max }$ & Best generation cost (\$/h) \\
\hline 3TA & $1,1.3$ & 0.6 & $1,0.5$ & $0.35,-0.35$ & $3,125.5$ \\
\hline 3TB & $1.1,2$ & 0.4 & $0.8,0.4$ & $0.30,-0.30$ & $3,127.7$ \\
\hline 3TC & $\mathbf{1 . 2 , 1}$ & $\mathbf{0 . 3}$ & $\mathbf{0 . 7 , 0 . 3}$ & $\mathbf{0 . 2 5}, \mathbf{- 0 . 2 5}$ & $3, \mathbf{1 2 5 . 3}$ \\
\hline 3TD & $2,1.6$ & 0.2 & $0.75,0.35$ & $0.20,-0.20$ & $3,128.2$ \\
\hline 3TE & 2,2 & 0.1 & $0.9,0.4$ & $0.10,-0.10$ & $3,126.1$ \\
\hline
\end{tabular}




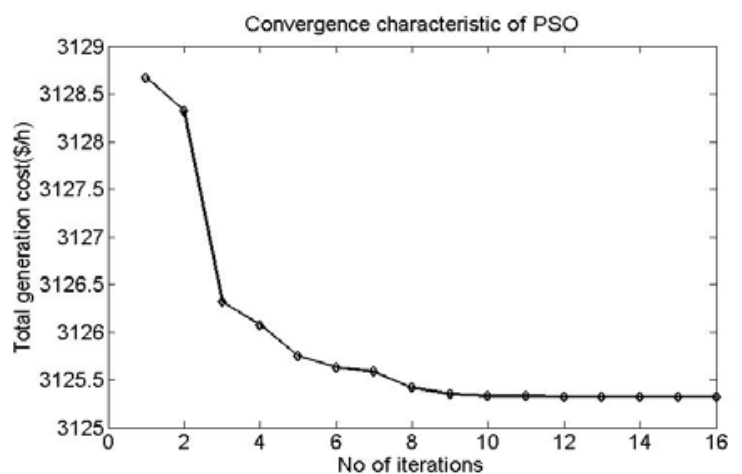

Figure 10. Convergence characteristic of PSO

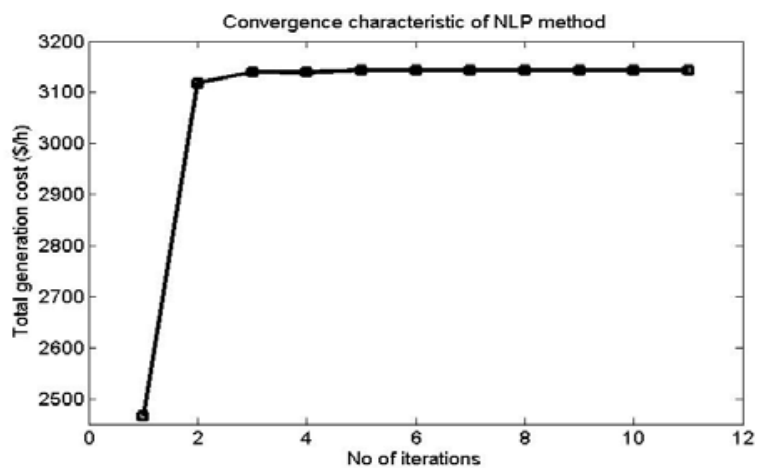

Figure 11. Convergence characteristic of NLP

\section{Conclusion}

This paper has proposed PSO based algorithm to maximize social benefit with/without using TCSC. The major outcome of the paper can be summarized as follows (1) Solutions obtained from PSO method are superior to non-linear programming method in solving cost minimization OPF problem. (2) Optimally placed TCSC by PSO could significantly reduce total generation cost, real and reactive power losses, total wheeling charges, real and reactive power spot prices and could increase social benefit of market participants and secure bilateral transaction. (3) When optimized output parameters obtained from PSO are used as initial staring points in interior point method to obtain spot prices, then it gives better results than those obtained from their default settings used in MATPOWER. (4)PSO outperforms interior point method in obtaining secure bilateral transaction matrix. So the proposed PSO based algorithm can be applied at planning stage while installing TCSC and it is also suitable for on line application at the energy management centre due to its robust convergence characteristic.

\section{References}

Acharya Naresh and Mithulananthan N., 2007. Influence of TCSC on congestion and spot price in electricity market with bilateral contract, Electric power system research, Vol. 77, pp. 1010-1018.

Cai L.J., Erlich I. and Stamtsis G., 2004. Optimal choice and allocation of FACTS devices in deregulated electricity market using genetic algorithm, in:proc.of IEEE PES power system Conf. and exposition, USA, pp. 10-13.

Dutta Sudipta and Singh S.P., 2008. Optimal rescheduling of generators for congestion management based on particle swarm optimization, IEEE Trans. Power Syst., Vol. 23, No. 4, pp. 1560-1568.

Gerbex S, Cherkaoui R and Germond A.J., 2001. Optimal location of multi-type FACTS devices in a power system by means of Genetic algorithms, IEEE Trans. Power Syst. 16 (3) (2001) pp. 537-544.

Hingorani N.G. and Gyugyi L., 2000. Understanding FACTS Concepts and Technology of Flexible AC Transmission Systems, IEEE press, ISBN-0-7803-3455-8.

Jirapong Peerapol and Ongsakul Weerakorn, 2007. Optimal placement of multi-type FACTS devices for total transfer capability enhancement using hybrid evolutionary algorithm, Electr. Power Components and Syst., Vol.35, pp. 981-1005.

Kennedy J. and Eberhart R, 1995. Particle swarm optimization, in: Proceedings of the IEEE International Conf. on Neural Networks, pp. 1942-1948.

Lima F.G.M., Galiana F.D., Kockar Ivana and Munoz J., 2003. Phase shifter placement in large-scale systems via mixed integer linear programming, IEEE Trans. Power Syst., Vol. 18, No. 3, pp. 1029-1034.

Lin Whei-Min, Chen shi-Jaw and Su Yuh-Sheng., 2000. An application of interior-point based OPF for system expansion with FACTS devices in a deregulated environment, in Proc. IEEE conference (0-7803-6338-8/00/\$10).

Luenberger D.G., 1995. Microeconomics theory. New York: McGraw-Hill, p. 187.

Murtagh B.A. and Saunders M.A., MINOS 5.5 user's guide. Stanford Univ. Systems, optimization laboratory tech. Rep. SOL 8320R.

Park J.B., Lee K.S., Shin J.R. and Lee K.Y., 2005. A particle swarm optimization for economic dispatch with nonsmooth cost functions, IEEE Trans. Power Syst., Vol. 20, No. 1, pp. 34-42.

Rashidinejad M. et al, 2008. ATC enhancement using TCSC via artificial intelligent techniques, Electric Power System Research, Vol. 78, 2008, pp. 11-20.

Sharma Ashwani kumar, 2006. Optimal number and location of TCSC and loadability enhancement in deregulated electricity markets using MINLP, International Journal of Emerging Electric Power Systems, Vol. 5, No. 1. 
Sharma Ashwani kumar and Chanana S., 2009. New secure bilateral transaction determination and study of pattern under contingencies and UPFC in competitive hybrid electricity markets, Electrical power and energy systems, Vol. 31, pp. 23-33.

Singh J.G., Singh S.N.and Srivastava S.C., 2007. An approach for optimal placement of static VAR compensators based on reactive power spot price, IEEE Trans. Power Syst., Vol. 22, No.4, pp. 2021-2029.

Verma K. S.and Gupta H.O., 2006. Impact on real and reactive power pricing in open power market using unified power flow controller, IEEE Trans. Power Syst., Vol. 21. No 1, pp. 365-371.

Wang H., Murillo-sanchez C.E., Zimmerman R.D. and Thomas R.J., 2007. On computational issues of market-based optimal power flow, IEEE Trans. Power Syst., Vol. 22, No.3, pp. 1185-1193.

Xiao Ying, Song Y.H., Liu Chen-Ching and Sun Y.Z., 2003. Available transfer capability enhancement using FACTS devices, IEEE Trans. Power Syst., Vol. 18. No.1, pp. 305-312.

Xie K. and Song Y.H., 2001. Power market oriented optimal power flow via an interior point method, IEE Proc.-Gener. Transm. Distrib., Vol. 148, No. 6, pp. 549-556.

Yang Guang Ya et al, 2007. TCSC allocation based on line flow based equations via mixed integer programming, IEEE Trans. Power Syst. Vol. 22, No 4, pp.2262-2269.

Yu X., Singh C, Jakovljevic S, Ristanovic D and Huang G., 2003. Total transfer capability considering FACTS and security constraints, in: Proc. IEEE Conference (0-7803-8110-6/03/\$17.00).

Yu Zuwei and Lusan D., 2004. Optimal placement of FACTS devices in deregulated systems considering line losses, Electrical power and energy systems, vol. 26, pp. 813-819.

Zhao B., Guo C.X. and Cao Y.J., 2005. A multiagent-based particle swarm optimization approach for optimal reactive power dispatch, IEEE Trans. Power Syst., Vol. 20, No. 2, pp. 1070-1078.

Zimmerman R.D. and Sanchez C.E.Murillo, MATPOWER, a Matlab power system simulation package, Version 3.2, 2007. [Online] . Available http:// www. pserc.cornell.edu/matpower/.

\section{Biographical notes:}

S. K. Joshi received M. E. and Ph.D. from the M. S. University of Baroda and Indian Institute of Technology Kanpur, India, respectively. He is a Professor in the Department of Electrical Engineering, M. S. University of Baroda, India. His research interests include power system restructuring, power system optimization \& control, voltage stability analysis and Artificial intelligence applications to power system. He is a member of IEEE.

K. S. Pandya received M.E. from L.D.College of Engineering, Gujarat, INDIA. Currently, he is pursuing Ph.D. from M.S.university of Baroda, INDIA. He is an Associate Professor in the Department of Electrical Engineering, Charotar Institute of Technology, CHANGA, INDIA. His current area of research includes power system restructuring, power system optimization, FACTS devices and artificial intelligence applications to power system. He is a member of IEEE.

Received November 2009

Accepted March 2010

Final acceptance in revised form March 2010 